\title{
Constitutive Investigation on Viscoelasticity of PolyVinyl Butyral: Experiments Based on Dynamic Mechanical Analysis Method
}

\author{
Bohan Liu, Jun Xu, and Yibing Li \\ State Key Laboratory of Automotive Safety \& Energy, Department of Automotive Engineering, Tsinghua University, \\ Beijing 100084, China \\ Correspondence should be addressed to Bohan Liu; liubh09@mails.tsinghua.edu.cn and Jun Xu; xujun06@mails.tsinghua.edu.cn
}

Received 26 November 2013; Accepted 22 January 2014; Published 11 March 2014

Academic Editor: Hazizan Md Akil

Copyright (C) 2014 Bohan Liu et al. This is an open access article distributed under the Creative Commons Attribution License, which permits unrestricted use, distribution, and reproduction in any medium, provided the original work is properly cited.

PolyVinyl Butyral (PVB) film is now widely used in automotive industry and architectures serving as the protective interlayer. The dynamic modulus of PVB is measured through systematic experiments based on Dynamic Mechanical Analysis (DMA) method at various temperatures, heating rates, and vibration frequencies. Further, viscoelasticity of PVB influenced by time and temperature is systematically studied. Fitted empirical formulas describing the relationship between glass transition temperature and frequency, as well as the heating rate of PVB, are established. The master curve of PVB at $293 \mathrm{~K}$ is suggested based on the experiment data as to express the dynamic modulus variation at various frequencies in a wider range. Constitutive behavior of PVB is then analyzed based on Generalized Maxwell (GM) model and Fractional Derivative (FD) model, respectively. It is shown that PVB has higher efficiency of energy dissipation in its high energy absorption state, while both fifth-order GM model and FD model can characterize the viscoelasticity of PVB at glassy transition area. Results may offer useful fundamental experimental data and important constitutive characteristics of PVB and shed lights on further studies on viscoelasticity behavior of PVB and energy mitigation ability of laminated glass.

\section{Introduction}

In traffic accidents, human heads usually suffer from serious injury by impacting on windshield, and this situation frequently occurs in pedestrians or cyclists involved accident [1-3]. PolyVinyl Butyral (PVB), a typical polymer material, is the prior choice of the interlayer material in laminated windshield with its excellent optical clarity, adhesive property, toughness, and flexibility [4-6]. Open literatures have pointed out that an effective way to reduce the head injury is to enhance the protection of the laminated windshield $[7,8]$. On the other hand, it is of great need to design and build ultrastrong laminated glass panels in architecture such to maintain integrity subject to impact or blast. The polymeric interlayer provides the fundamental protection during the impact mainly due to its viscoelasticity $[9,10]$. Therefore, investigating the mechanical viscoelasticity of PVB interlayer becomes a great interest to the relevant fields.
Several experimental attempts have been made to investigate PVB which moves the first step towards understanding the mechanical behavior of PVB material. For instance, Iwasaki et al. [11] obtained the stress-strain curves of PVB in tension tests at different strain rates from $0.0067 / \mathrm{s}$ to $118 /$ s. It proved PVB as a highly rate dependent material which is partially related to its viscoelasticity. Further, Xu et al. [12] gained dynamic stress-strain curves of PVB at different strain rates from $800 / \mathrm{s}$ to $4500 / \mathrm{s}$ under compression loading via Split Hopkinson Pressure Bar (SHPB) [13, 14] method. The rate dependent characteristic of PVB is also found in high strain rate range and further a rate dependent constitutive model of PVB is fitted by Liu et al. [4] based on both quasistatic and dynamic experiment data. Hooper et al. [15] obtained the mechanical behavior of PVB at small $(<0.1 \%)$ and large $(>200 \%)$ strains over a range of strain rates to provide data for the development and validation of constitutive models. Furthermore, Callewaert et al.s [16] 
study focused on the viscoelasticity of the interlayer material of laminated glass, that is, PVB and SentryGlas (SG). Creep and relaxation tests in different temperatures are carried out, and it is proved that interlayer material has a major influence on the overall mechanical behavior of laminated glass [7]. However, Current research on PVB material are not available in terms of the systematic viscoelasticity behavior, while time and temperature effect on PVB's constitutive model is lack of consideration. Any attempts to further investigate the mechanical behavior of laminated glass and to enhance the safety of windshield and architectural glass should be only based on a thorough understanding on the viscoelasticity behavior of polymer interlayer material, which is our fundamental motivation for this research.

The widely accepted experimental method to directly study the viscoelasticity of polymer is Dynamic Mechanical Analysis (DMA) method [17-19]. For example, the viscoelasticity of asphalt [20] and block poly(propylene carbonatecyclohexyl carbonate) (PPC-PCHC) [21] are studied based on Time-Temperature-Superposition (TTS) principle. These studies have shown the capability of DMA method to fully reveal the viscoelasticity properties of materials.

Thus, in present paper, we focus on the PVB's dynamic viscoelasticity behavior and time-temperature effect. DMA method is employed to conduct the time scanning and temperature scanning test, which systematically investigates the viscoelasticity and time-temperature coupling effect of PVB material in a parametrical way. Based on the gained viscoelasticity behavior, Willianms-Landel-Ferry (WLF) [22] equation, which expresses the shift factor in different temperatures, is used to fit the master curve of PVB. Generalized Maxwell (GM) model and Fractional Derivative (FD) model are employed to describe the viscoelastic constitutive behavior.

\section{Dynamic Mechanical Analysis (DMA) Experiments}

2.1. Experimental Setup. DMA 2980 Dynamic Mechanical Analyzer (produced by TA Instrument Corporation, CA, USA) is employed. Loading and displacement dpi of the device are $10^{-4} \mathrm{~N}$ and $1 \mathrm{~nm}$, respectively, and modulus accuracy is in $\pm 1 \%$. According to the experiment requirement, the dimension of testing specimen is set as $5 \mathrm{~mm}$ in width and $0.76 \mathrm{~mm}$ in thickness, which is the most widely used thickness in current PVB application in laminated windshield [23].

The DMA system is set as shown in Figure 1. Two sides of PVB specimen are clamped with the distance of $20 \mathrm{~mm}$. The up-clamped end is fixed while the down-clamped end is moving enduring experiment. During the test, static and dynamic forces are applied simultaneously: static force keeps the specimen a constant strain 0.01, while dynamic force makes specimen a sinusoidal vibration with the amplitude of $20 \mu \mathrm{m}$. Viscoelasticity of PVB leads to a phase lag for strain (deformation) so the stress and strain can be expressed as [22]

$$
\begin{gathered}
\sigma(t)=\sigma_{0} \sin \omega t, \\
\varepsilon(t)=\varepsilon_{0} \sin (\omega t+\delta),
\end{gathered}
$$

where $\sigma(t), \varepsilon(t)$ stand for stress and strain at the time of $t$, respectively. $\sigma_{0}$ and $\varepsilon_{0}$ are the amplitude of stress and strain, respectively. $\omega$ is the vibration angular velocity and $\delta$ is the phase lag.

Furthermore, phase relationship between stress and strain can be used to calculate the plural elastic modulus as [22]

$$
\begin{aligned}
E^{*}=\frac{\sigma}{\varepsilon}=E^{\prime}+i E^{\prime \prime} & =E^{*} \cos \delta+i E^{*} \sin \delta, \\
\tan \delta & =\frac{E^{\prime \prime}}{E^{\prime}}
\end{aligned}
$$

where $E^{*}$ is plural elastic modulus, $E^{\prime}$ stands for storage modulus, and $E^{\prime \prime}$ represents loss modulus. $\delta$ denotes the phase angle.

2.2. Experiment Design Matrix. In order to systematically investigate the influence of time and temperature on the viscoelasticity of PVB material, frequency and temperature scanning test are carried out in different conditions separately.

2.2.1. Frequency Scanning Test. Considering the frequency range of the instrument $(0.01 \mathrm{~Hz}$ to $200 \mathrm{~Hz})$, twenty frequency sampling points in range from $0.01 \mathrm{~Hz}$ to $80 \mathrm{~Hz}$ are selected in each group of frequency scanning test. According to [24], $0.5 \sim 25 \mathrm{~Hz}$ is the main vibration frequency range of driving automotive caused by road irregularities, while 1st phase torsional frequency of vehicle body-in-white and an important indicator for the strength of the vehicle frame is $24.85 \mathrm{~Hz}$ [25]. Hence, frequency scanning range covers the PVB's application in automotive windshield vibration. In temperature range from $223 \mathrm{~K}$ to $323 \mathrm{~K}$, in total eleven temperature sampling points are selected (take one point every $10 \mathrm{~K}$ ) to obtain the frequency scanning data.

2.2.2. Temperature Scanning Test. Temperature scanning range is from $223 \mathrm{~K}$ to $323 \mathrm{~K}$, covering nearly all the possible ambient temperature range where PVB is applied in automotive windshield interlayer. Six frequency sampling points $(1 \mathrm{~Hz}, 2 \mathrm{~Hz}, 5 \mathrm{~Hz}, 10 \mathrm{~Hz}, 20 \mathrm{~Hz}$, and $50 \mathrm{~Hz})$ are selected to obtain the temperature scanning data at heating rate $5 \mathrm{~K} / \mathrm{min}$. Meanwhile, at frequency $10 \mathrm{~Hz}$, five heating rate sampling points $(2.5 \mathrm{~K} / \mathrm{min}, 5 \mathrm{~K} / \mathrm{min}, 7.5 \mathrm{~K} / \mathrm{min}, 10 \mathrm{~K} / \mathrm{min}$, and $12.5 \mathrm{~K} / \mathrm{min}$, resp.) are chosen. As aforementioned, ten sampling points of both frequency and heating rate are selected in the temperature range.

In total, there are 21 groups of experiments in total carried out in this paper.

\section{Experiment Results}

Experimental curves describing storage modulus $E^{\prime}$, loss modulus $E^{\prime \prime}$, and tangent value of phase angle $\tan \delta$ varying with temperature and frequency are obtained via dynamic scanning tests. Note that at the frequency of $80 \mathrm{~Hz}$, experiment result deviates from the curves due to the resonating 


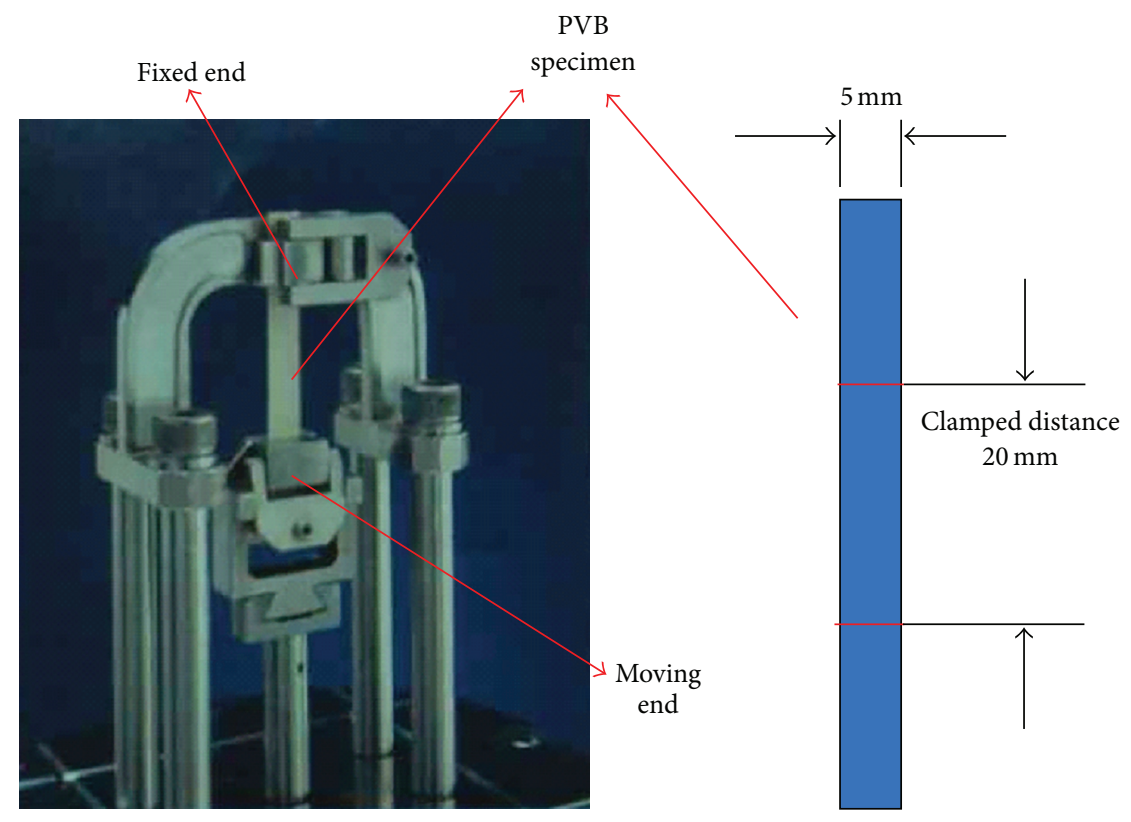

FIgure 1: Illustration of DMA 2980 Dynamic Mechanical Analyzer and dimension of PVB specimen.

between instrument and specimen so points at frequency $80 \mathrm{~Hz}$ are removed.

\subsection{Frequency Dependency of Dynamic Modulus. Dynamic} modulus-frequency curves in different temperatures obtained by frequency scanning test are illustrated together in Figure 2. As shown in Figure 2, one may observe a significant difference for the dynamic modulus in different temperature ranges. The values of storage and loss modulus of PVB are both relatively high once the temperature is below $273 \mathrm{~K}$. When the temperature is between $273 \mathrm{~K}$ and $323 \mathrm{~K}$, $\log \left(E^{\prime}\right)$ and $\log \left(E^{\prime \prime}\right)$ increases linearly with $\log (f)$. If the temperature rises above $323 \mathrm{~K}$, the value of storage modulus maintains unchanged in a lower level (i.e., lower than $10 \mathrm{MPa}$ ). At lower temperature, PVB is in glassy state [26] by exhibiting higher dynamic modulus where PVB shows to be "hard" and "brittle" and the dynamic modulus is slightly affected by temperature and frequency. As temperature increases, the dynamic modulus of PVB begins to decreases rapidly and a transition from glassy to rubbery state occurs at the threshold value $T_{1}=273 \mathrm{~K}$. During this transition, an increase in temperature has the equivalent effect on PVB as a decrease in frequency, and this phenomenon is dominated by TTS principle in polymer material. Once temperature goes beyond another threshold value, that is, $T_{2}>323 \mathrm{~K}$, the transition from glassy to rubbery state ends and PVB changes into rubbery status. So the dynamic modulus of PVB decreases to lower level where PVB exhibits to be "soft" and "ductile".

3.2. Temperature Dependency of Dynamic Modulus. Dynamic modulus-temperature curves at different frequencies obtained by temperature scanning test are illustrated together in Figure 3. When the temperature is below $T_{1}$, $E^{\prime}$ is about $1000 \mathrm{MPa}$ while $E^{\prime \prime}$ is about $100 \mathrm{MPa}$. In this temperature range, frequency had little effect on dynamic modulus of PVB. When the temperature is above $T_{1}, \log \left(E^{\prime}\right)$ and $\log \left(E^{\prime \prime}\right)$ decrease significantly as the temperature increase. At lower temperature, the thermal energy is not enough to reach the threshold energy to readjust the molecular chain segment; thus the chain segment remains in a "frozen" state. By fixing the molecular chain segment in the microscale, the material itself shows a higher dynamic modulus. As temperature increases, thermal energy reaches the readjusting energy barrier of molecular chain segment, and PVB translates from glassy to rubbery state gradually. On the other hand, at higher frequency, molecular chain segment has shorter time to response to the applied stress and have no time to readjust. Stress can only make the change of distance between molecules; therefore, similar to the case at lower temperature, it is considered that molecular chain segment is in "frozen" state at higher frequency, presenting to be higher dynamic modulus. As frequency decreases, time is enough for molecular chain segment to readjust, which can relax the local strain. Furthermore, PVB relaxed into a lower energy conformation shows a lower dynamic modulus under vibration [22].

3.3. Glass Transition Temperature. In glass transition temperature $t_{g}, \tan \delta$ reached to its maximum value. In order to investigate the influence factors of glass transition temperature, $\tan \delta$-temperature curves in different frequencies and heating rates are illustrated in Figure 4. When temperature is above $T_{1}, \tan \delta$ begins to increases rapidly and PVB enters the glassy transition region and begin to translate from glassy state to rubbery state. As the scanning frequency and heating rate increases, the glass transition region of PVB translate 


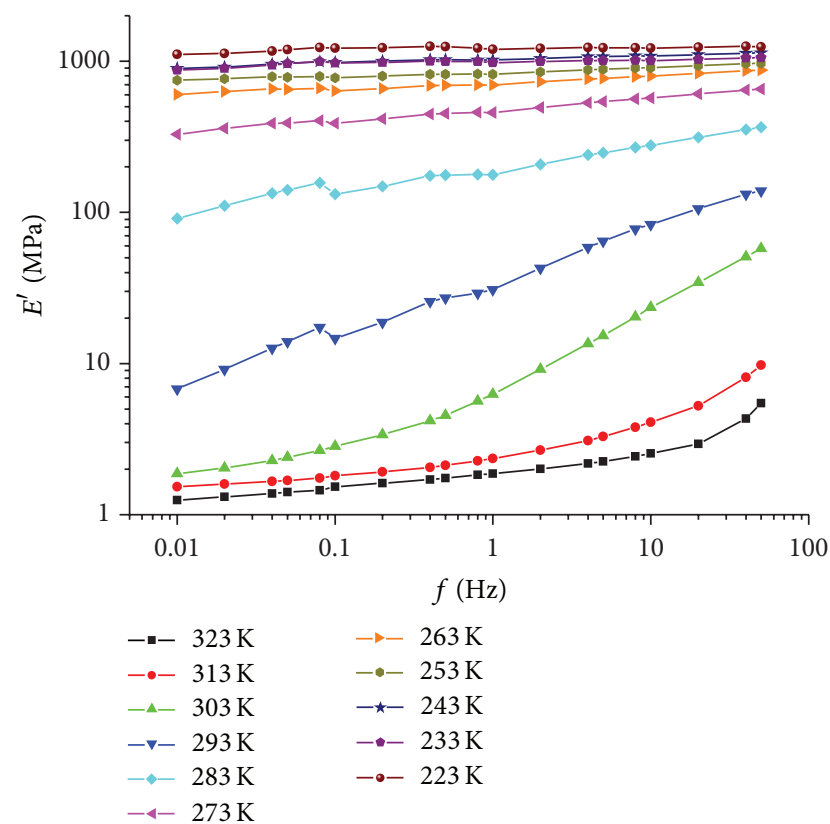

(a)

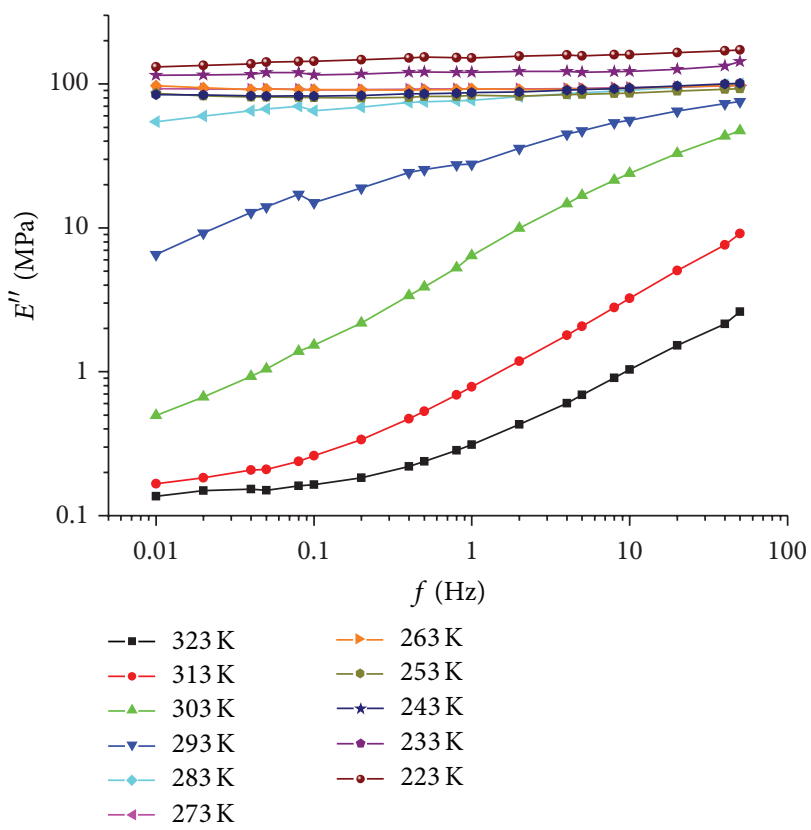

(b)

FIGURE 2: Dynamic modulus variation as to various frequency from $0.01 \mathrm{~Hz}$ to $50 \mathrm{~Hz}$ at temperatures from $223 \mathrm{~K}$ to $323 \mathrm{~K}$; (a) storage modulus; (b) loss modulus.

to higher temperature zone and glass transition temperature increases gradually.

From the $t_{g}$-frequency curve at heating rate $5 \mathrm{~K} / \mathrm{min}$ and $t_{g}$-heating rate curve at frequency $10 \mathrm{~Hz}$, it is shown that $t_{g}$ increases with frequency and heating rate in a linear relationship. Here we define the room temperature $T_{0}=$ $293 \mathrm{~K}$ and heating rate $v_{0}=5 \mathrm{~K} / \mathrm{min}$ as the reference values. Considering that the 1st phase torsional frequency of vehicle

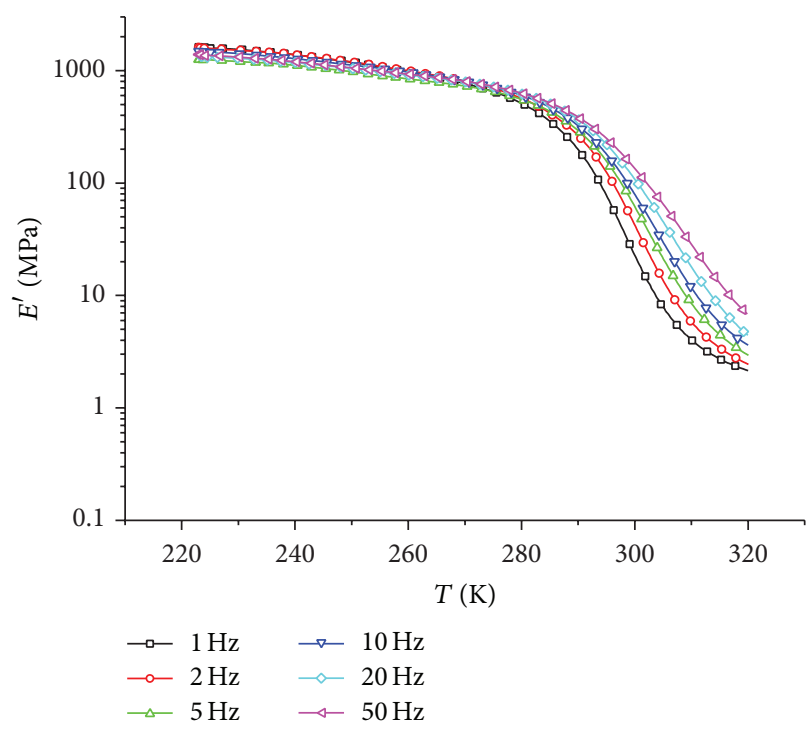

(a)

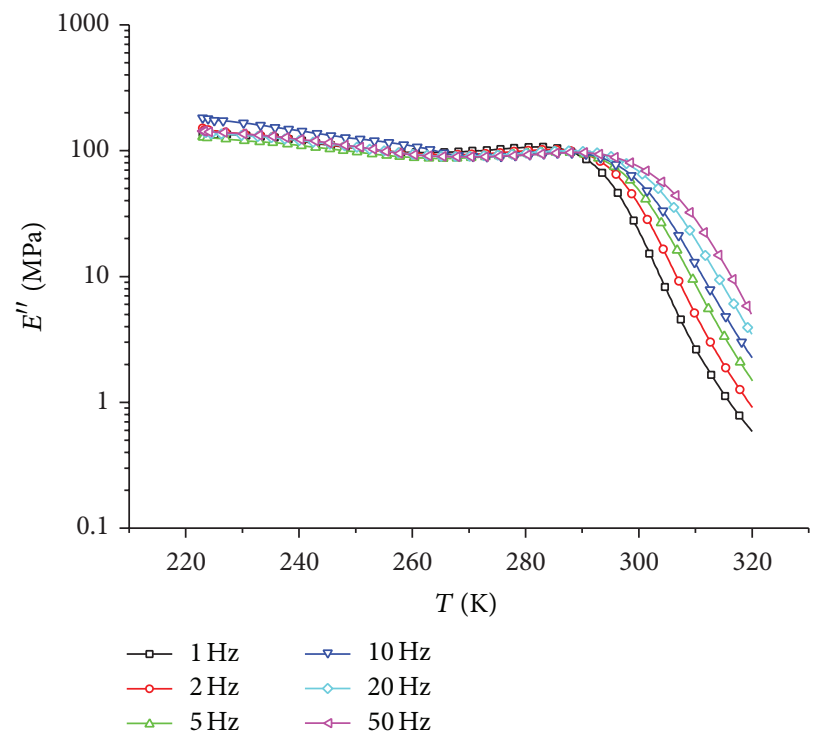

(b)

Figure 3: Dynamic modulus variation as to various temperature from $223 \mathrm{~K}$ to $323 \mathrm{~K}$ at frequency from $1 \mathrm{~Hz}$ to $50 \mathrm{~Hz}$; (a) storage modulus; (b) loss modulus.

body-in-white is $24.85 \mathrm{~Hz}$ [25], the reference frequency is defined as $f_{0}=25 \mathrm{~Hz}$. With the definition of normalized variable $\Omega=T / T_{0}, \Psi=f / f_{0}$, and $\Xi=v / v_{0}$, the equation $\Omega=F(\Xi, \Psi)$ to describe the relation between $t_{g}$, heating rate, and scanning frequency, can be fitted as

$$
\Omega=(0.046 \lg (\Psi)+2.076) \times(0.006 \Xi+0.506) .
$$

Fitting results compared with the experimental data are shown in Figure 5. It is shown that at the heating rate from 0 to $12.5 \mathrm{~K} / \mathrm{min}$ and scanning frequency from $1 \mathrm{~Hz}$ to $50 \mathrm{~Hz}, t_{g}$ varies from $298 \mathrm{~K}$ to $319 \mathrm{~K}$. 


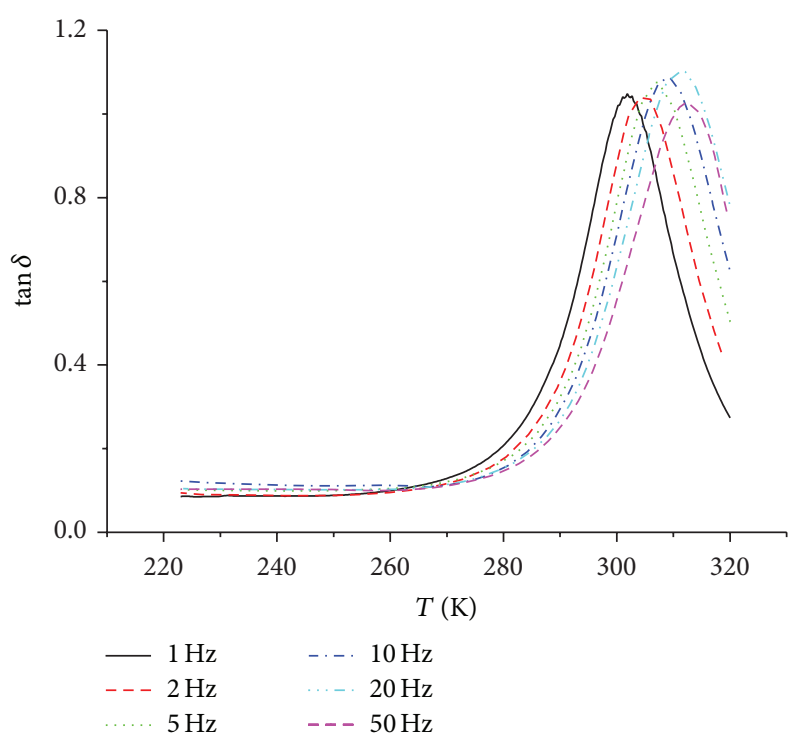

(a)

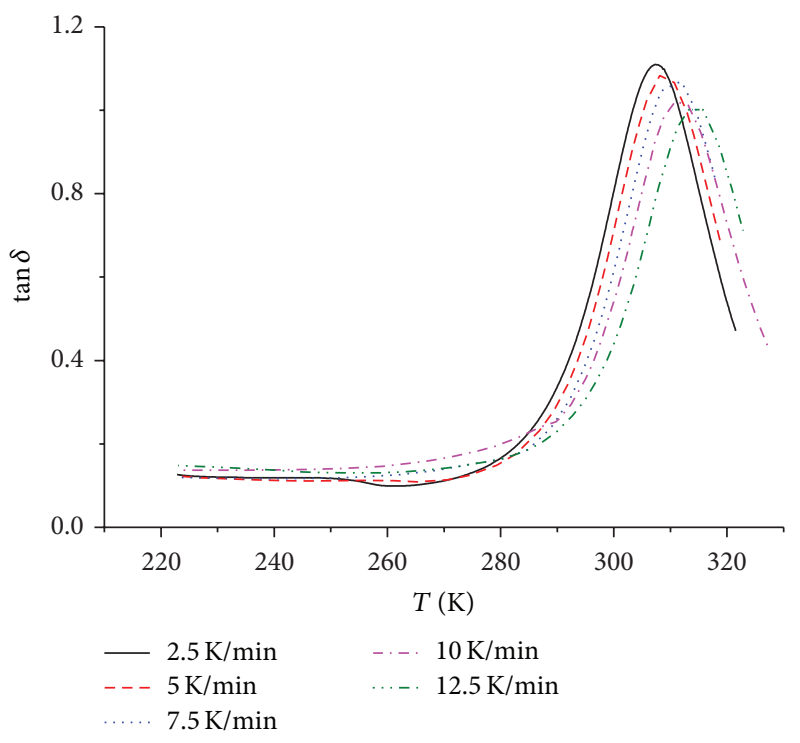

(b)

FIGURE 4: $\tan \delta$ variation as to various temperature from $223 \mathrm{~K}$ to $323 \mathrm{~K}$ at frequency from $1 \mathrm{~Hz}$ to $50 \mathrm{~Hz}$ and heating rate from $2.5 \mathrm{~K} / \mathrm{min}$ to $12.5 \mathrm{~K} / \mathrm{min}$; (a) at various frequencies; (b) at various heating rates.

\section{Discussions}

4.1. Energy Dissipation. When molecular chain segment is in "frozen" state, chain segment will not adjust under external stress and cannot dissipate energy by friction and relative movements. In the certain environment condition, when temperature increases to $t_{g}$, friction produced by relatively move between chain segment is in maximum and ratio of energy dissipation (i.e., loss modulus) and energy storage (i.e., storage modulus) reaches to the peak value [22]. When temperature is above $t_{g}$, chain segment begins to move freely, and the friction declines while energy dissipation decreases.

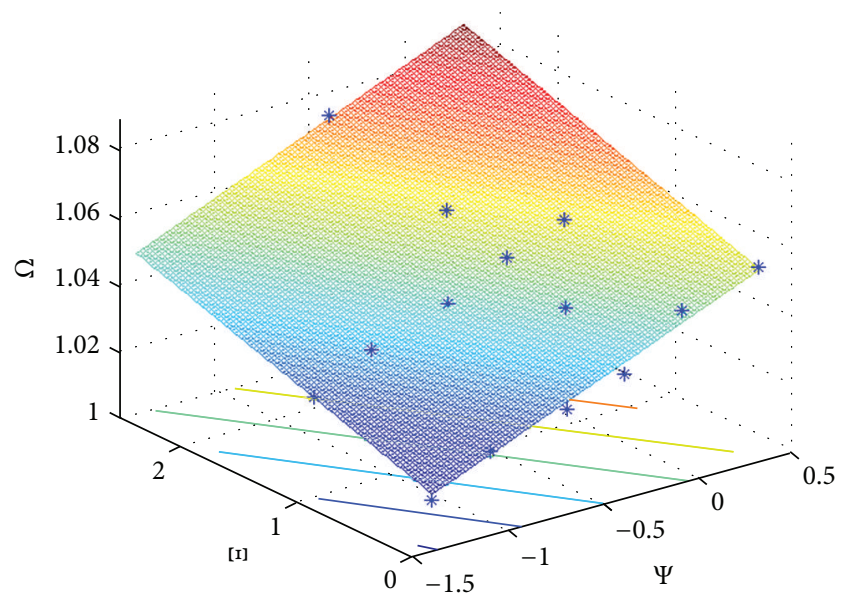

FIGURE 5: Fitting result of glass transition temperature at frequency from $1 \mathrm{~Hz}$ to $50 \mathrm{~Hz}$ and heating rate from $2.5 \mathrm{~K} / \mathrm{min}$ to $12.5 \mathrm{~K} / \mathrm{min}$ compared with experimental data in $3 \mathrm{D}$ graph.

According to the analysis Section 3.3, when heating rate is at $5 \mathrm{~K} / \mathrm{min}, t_{g}$ of PVB is between $302 \mathrm{~K}$ and $314 \mathrm{~K}$. In Figure 4, we choose the curve segment at $\tan \delta>0.5$, corresponding to an extending temperature range of $t_{g}$, that is, 293 323 K. In this temperature range, efficiency of energy dissipation is in the highest level and PVB is suitable to apply as an energy absorption component [23]. We considered PVB to be in a high energy absorption state in this temperature range. Relaxation and creep tests on PVB/SGP laminated glass carried out by other researchers [16] have obtained similar results. During the temperature range 293 323 K, the stiffness curve is significantly influenced by time and temperature range, both on the PVB laminated glass and SentryGlas (SG) laminated reinforced glass [27]. It is proved that interlayer polymer material plays a critical role in the energy mitigation behavior of laminated glass [7].

4.2. Validity of Time-Temperature-Superposition (TTS) Property. $E^{*}-\tan \delta$ curves of PVB in double logarithmic coordinate are illustrated in Figure 6. For thermorheological viscoelasticity material, it is clear that $E^{*}-\tan \delta$ curves in double logarithmic coordinate at different scanning temperatures can coincide in one continuous curve thus to meet the "thermorheological simplify" assumption [28]; thus, PVB is proven as a thermorheological simplified material.

Willianms-Landel-Ferry (WLF) equation can be employed based on "thermorheologic simplify" assumption expressed as follows [22]:

$$
\lg a_{T}=\frac{-C_{1}\left(T-T_{r}\right)}{C_{2}+T-T_{r}},
$$

where $C_{1}$ and $C_{2}$ are empirical constant, $T_{r}$ stands for reference temperature, $a_{T}$ refers for shift factor, and $T$ is actual temperature.

Furthermore, for PVB dynamic frequency scanning data, modulus-frequency curve at different temperatures can translate $\lg \alpha_{T}$ along the horizontal coordinate to generate the 


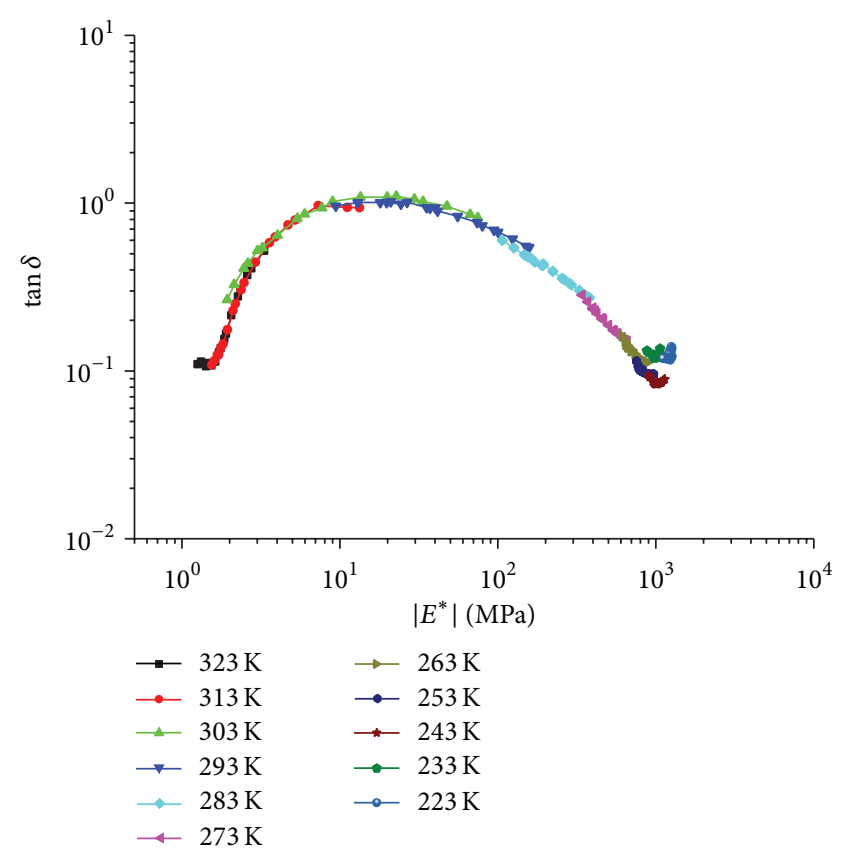

FIgURE 6: $\tan \delta-E^{*}$ curves at frequency from $0.01 \mathrm{~Hz}$ to $50 \mathrm{~Hz}$ at temperature from $223 \mathrm{~Hz}$ to $323 \mathrm{~Hz}$.

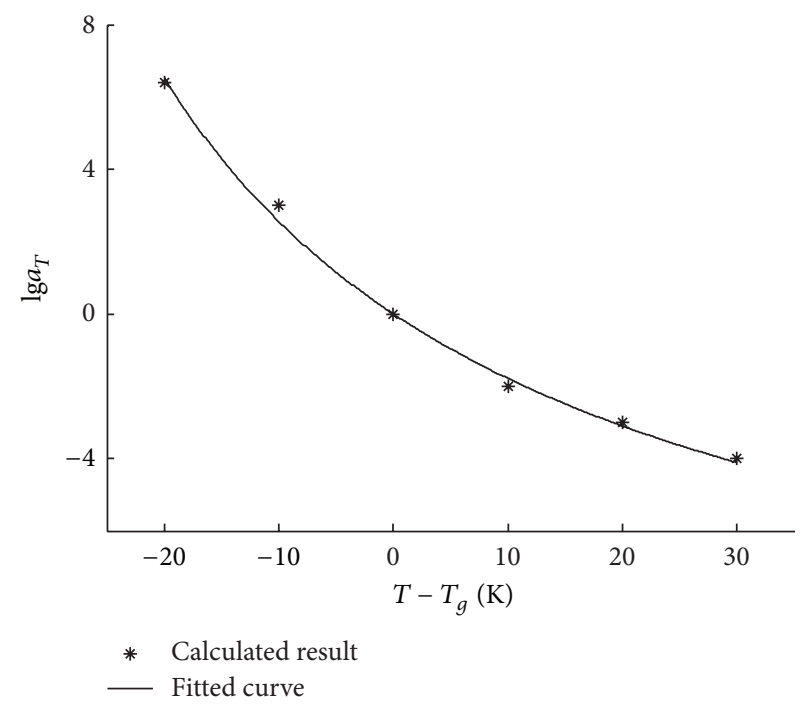

FIGURE 7: Fitted WLF curve compared with the calculated result at temperature $293 \mathrm{~K}$.

master curve of PVB. In this case, as a proved TTS applicable material, the viscoelasticity of PVB at the conditions beyond experiment device configuration may be obtained by extrapolating the experimental data at normal conditions. $T_{r}=$ $293 \mathrm{~K}$ is selected to be the reference temperature. With the logarithm of shift factor in different temperatures substituted in (4), WLF equation of PVB is fitted as follows:

$$
\lg a_{T}=\frac{-11.81\left(T-T_{r}\right)}{56.25+T-T_{r}}
$$

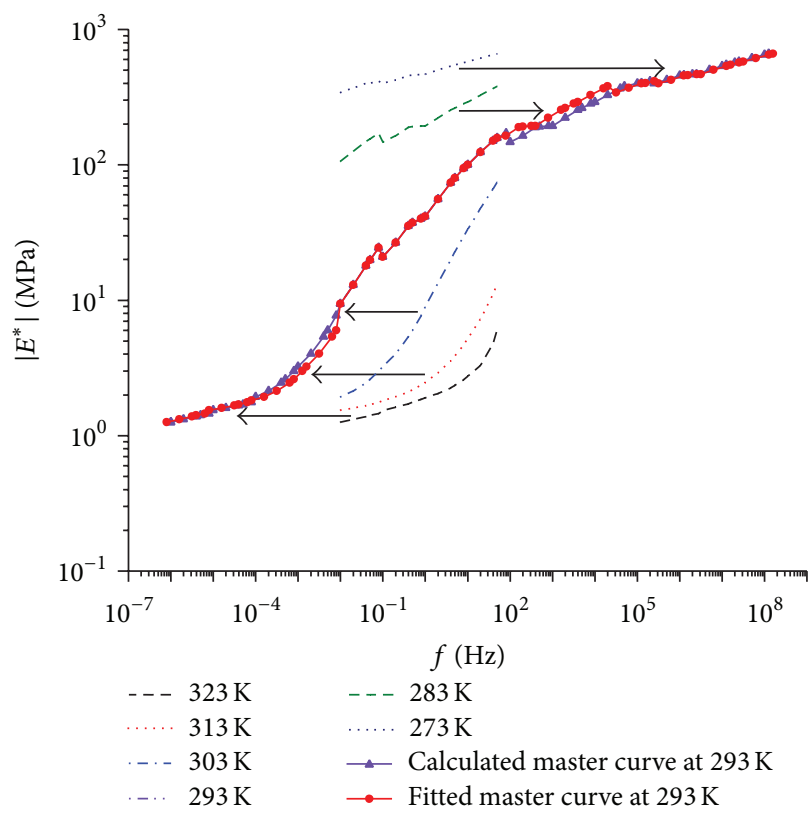

FIGURE 8: Calculated and fitted master curves at temperature $293 \mathrm{~K}$ compared with dynamic modulus-frequency curves at different temperatures.

The fitted curve and experiment data are analogous and with a good agreement, as shown together in Figure 7.

Based on the result of $\lg a_{T}$ and fitted WLF equation, master curve of dynamic modulus is plotted by comprising a group of shifted modulus-frequency curves at different temperatures, shown in Figure 8. It is verified that fitted master curve and calculated master curve are well resembled, and dynamic modulus in the frequency range of $10^{-6} \sim 10^{8} \mathrm{~Hz}$ can be described by the master curve.

From the result, dynamic mechanical characteristics of $\mathrm{PVB}$ in a more extensive frequency range are obtained without experiment and we can get the frequency threshold value at the boundary of different states in temperature $293 \mathrm{~K}$. As mentioned in Section 3.1, the transition from glassy to rubbery state occurs at a frequency threshold value $f_{1}$ and the transition finished and PVB changes into rubbery state at another frequency threshold value $f_{2}$. In either glassy or rubbery state, frequency had little effect on dynamic modulus of PVB, while within the glass transition region, dynamic modulus of PVB increases with frequency obviously, so we define $F_{1}$ and $F_{2}$ according to the slope of $\left|E^{*}\right|$. The 1st derivative of $\left|E^{*}\right|$ are illustrated in Figure 9. $\left(\partial\left|E^{*}\right| / \partial f\right)-f$ curve shows that in both glassy state and rubbery state, $\partial\left|E^{*}\right| / \partial f$ maintains in a lower value, while it is higher in glassy transition region. Threshold value $f_{1}$ and $f_{2}$ are obtained at $\left(\partial\left|E^{*}\right| / \partial f\right)=0.15$, where $f_{1}=3.17 \times 10^{-4} \mathrm{~Hz}$ and $f_{2}=7962 \mathrm{~Hz}$. In the same way, master curves and frequency threshold value at other temperatures can be obtained by experiment data and WLF equation to analyze the dynamic modulus influenced by frequency. 


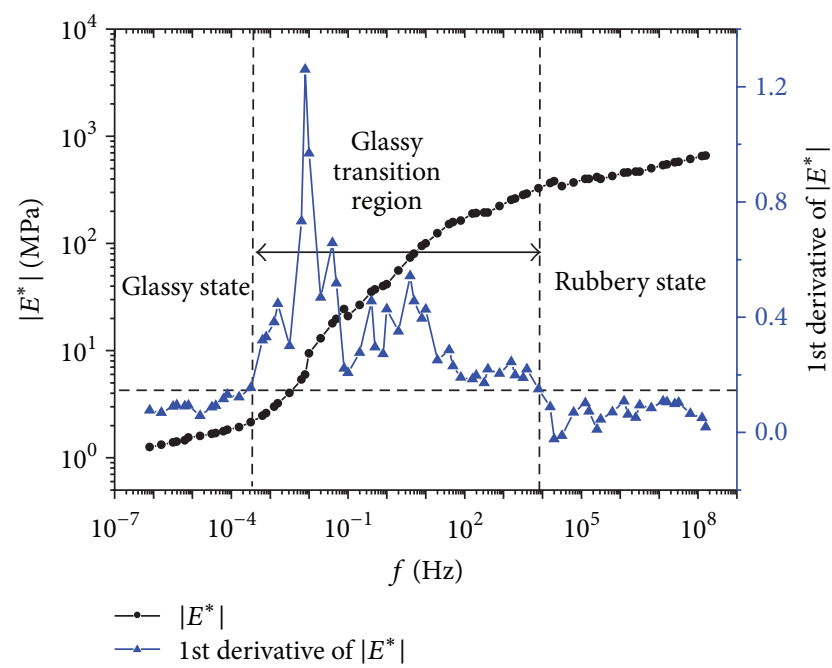

Figure 9: $\left|E^{*}\right|$ and $\partial\left|E^{*}\right| / \partial f$ variation as to various frequency from $10-6 \mathrm{~Hz}$ to $108 \mathrm{~Hz}$ at temperature $293 \mathrm{~K}$.

\subsection{Viscoelasticity Constitutive Relationship}

4.3.1. Generalized Maxwell (GM) Model. In Maxwell model, instantaneous elastic modulus $E$ is to characterize the response of spring while $\eta$ is to characterize the viscosity of dashpot [22]. GM model is comprised of several numbers of Maxwell components in parallel and its storage modulus and loss modulus is expressed as follows:

$$
\begin{aligned}
& E^{\prime}=\sum_{i=1}^{n} \frac{E_{i} \eta_{i}^{2}(2 \pi f)^{2}}{E_{i}^{2}+\eta_{i}^{2}(2 \pi f)^{2}}, \\
& E^{\prime \prime}=\sum_{i=1}^{n} \frac{E_{i}^{2} \eta_{i}(2 \pi f)}{E_{i}^{2}+\eta_{i}^{2}(2 \pi f)^{2}},
\end{aligned}
$$

where $E_{i}$ and $\eta_{i}$ are spring elastic constant and dashpot viscosity of group $i$ 's component in GM model. Without loss of generality, experiment data at reference temperature $293 \mathrm{~K}$ are employed to fit the GM model in third, fifth, and seventh orders. Fitting results compared with experiment data are illustrated in Figure 10.

It is considered that the model in the third order cannot describe the viscoelasticity of PVB at temperature $293 \mathrm{~K} \mathrm{accu}-$ rately and there is a significant deviation between fitting result and calculated result. Fifth- and seventh-order model can characterize the viscoelasticity more accurately. It is obvious that the accuracy of fit between fitting and experiment result increases with the order of model. To keep a balance between the number of parameters and the accuracy of GM model, fifth-order model is employed to fit the curve at temperature from $223 \mathrm{~K}$ to $323 \mathrm{~K}$, and the two correlation coefficients at every temperature are listed in Table 1. Fitting result are more accurate at temperature $293 \mathrm{~K}$ to $323 \mathrm{~K}$; the two correlation coefficients of storage modulus and loss modulus are above 0.99 .

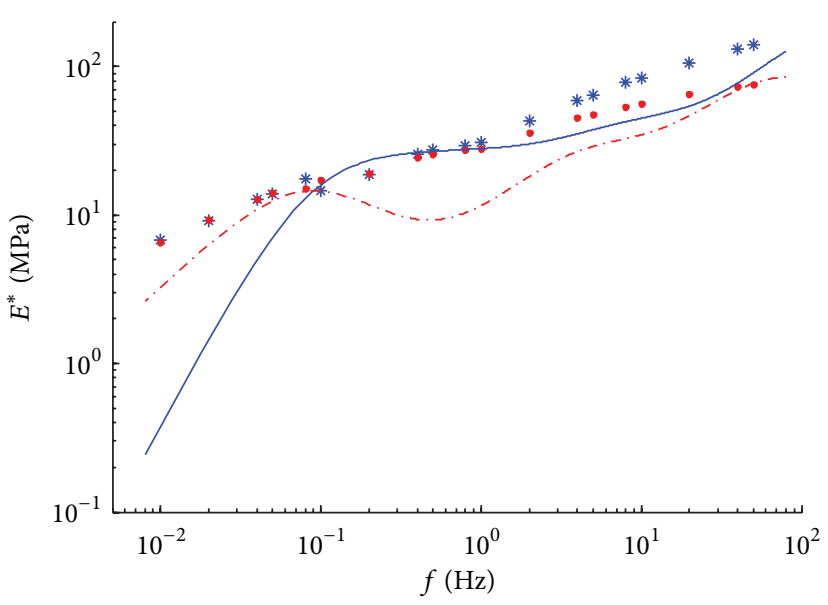

(a)

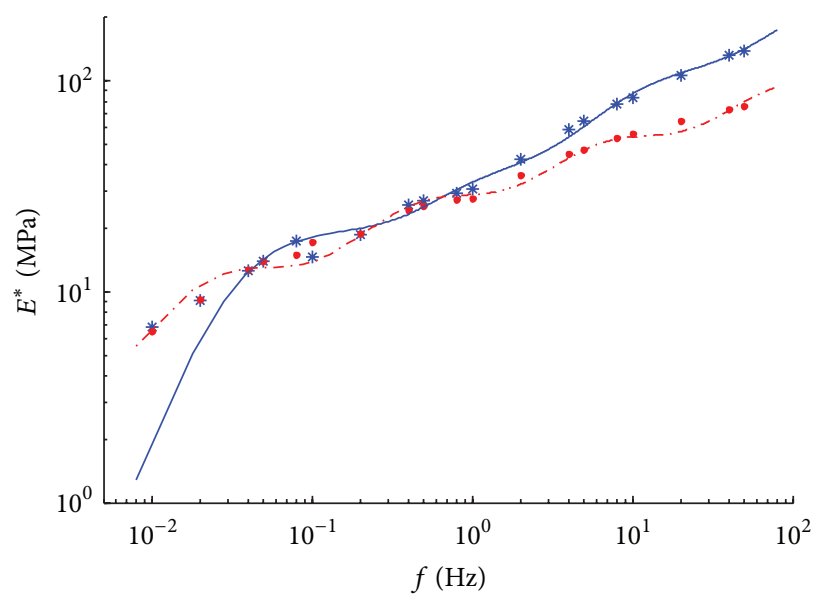

(b)

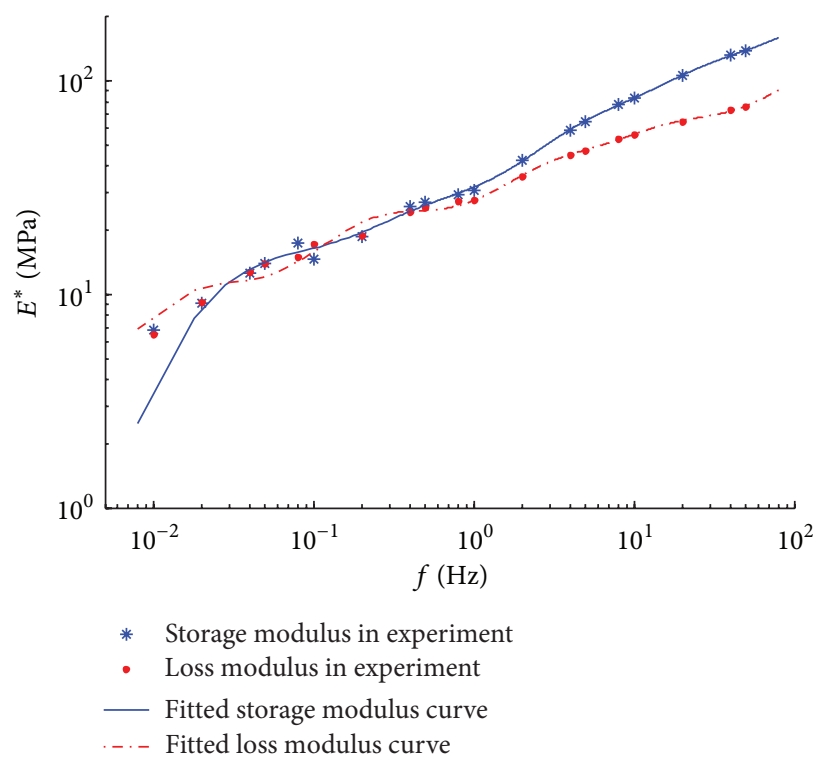

(c)

FIGURE 10: $\left|E^{*}\right|$ variation as to various frequency fitted by GM model compared with experiment results at temperature $293 \mathrm{~K}$; (a) in third order; (b) in fifth order; (c) in seventh order. 
TABLE 1: Correlation coefficients of fifth-order GM model.

\begin{tabular}{lccccccccccc}
\hline$T(\mathrm{~K})$ & 223 & 233 & 243 & 253 & 263 & 273 & 283 & 293 & 303 & 313 & 323 \\
\hline$R_{1}$ & 0.848752 & 0.927142 & 0.986776 & 0.98841 & 0.991388 & 0.994569 & 0.995426 & 0.998158 & 0.999917 & 0.999933 & 0.998882 \\
$R_{2}$ & 0.732023 & 0.613849 & 0.386564 & 0.348588 & 0.313536 & 0.075246 & 0.788232 & 0.994029 & 0.999926 & 0.999242 & 0.996427 \\
\hline
\end{tabular}

TABLE 2: Summary of fitting results of FD model.

\begin{tabular}{lccccc}
\hline$T(\mathrm{~K})$ & $\eta$ & $E$ & $r$ & $R_{1}$ & $R_{2}$ \\
\hline 223 & -3505.8 & 870.0281 & -0.07107 & 0.638793 & 0.967624 \\
233 & 516.3113 & -1153.24 & 0.017639 & 0.87531 & 0.822305 \\
243 & 718.745 & -2757.43 & 0.023908 & 0.981593 & 0.942977 \\
253 & -401.135 & 265.9266 & -0.01785 & 0.989054 & 0.827533 \\
263 & 324.6833 & -641.071 & 0.024936 & 0.987823 & 0.470596 \\
273 & 486.5962 & 2520.5 & 0.110543 & 0.994913 & 0.773588 \\
283 & 152.1968 & 4550.974 & 0.179521 & 0.992555 & 0.986505 \\
293 & 21.28383 & 706.851 & 0.396737 & 0.99876 & 0.994958 \\
303 & 3.458494 & 290.9311 & 0.567295 & 0.999437 & 0.999731 \\
313 & 1.408582 & -12.8328 & 0.270329 & 0.951102 & 0.996986 \\
323 & 0.615706 & -1.01452 & 0.05711 & 0.931893 & 0.993724 \\
\hline
\end{tabular}

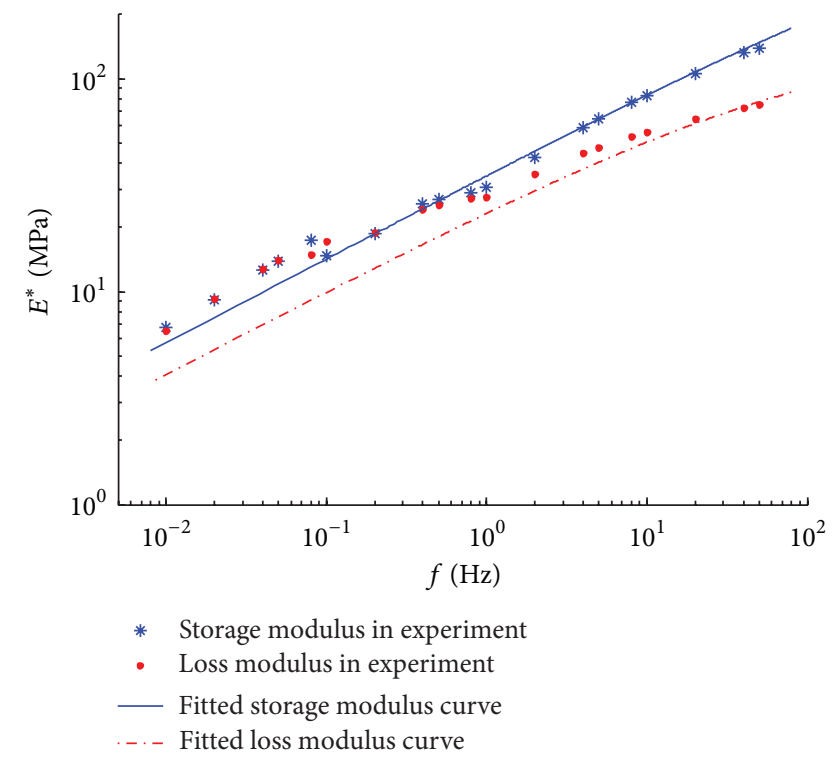

Figure 11: Curves fitted by Fractional Derivative model compared with experiment results at temperature $293 \mathrm{~K}$.

4.3.2. Fractional Derivative (FD) Model. For a pure elastic material, stress is proportional to strain rate, while the constitutive relationship of a viscoelastic material obeys $\sigma \sim$ $d^{r} \varepsilon / d t^{r}(0<r<1)$, which is to derive the FD model [20]. When $r=1$, Fractional Derivative model equals Maxwell model. Storage modulus and loss modulus can expressed as follows:

$$
\begin{aligned}
E^{\prime} & =\frac{\left(\eta^{2} / E\right)(2 \pi f)^{2 r}+\eta(2 \pi f)^{r} \cos (r \pi / 2)}{1+\left(\eta^{2} / E^{2}\right)(2 \pi f)^{2 r}+2(\eta / E)(2 \pi f)^{r} \cos (r \pi / 2)}, \\
E^{\prime \prime} & =\frac{\eta(2 \pi f)^{r} \sin (r \pi / 2)}{1+\left(\eta^{2} / E^{2}\right)(2 \pi f)^{2 r}+2(\eta / E)(2 \pi f)^{r} \cos (r \pi / 2)}
\end{aligned}
$$

Three parameters at different temperatures from $223 \mathrm{~K}$ to $323 \mathrm{~K}$ are fitted by the data obtained by the test at corresponding temperature, and three parameters and two correlation coefficients at each temperature are listed in Table 2 . As the temperature at $293 \mathrm{~K}$ and $303 \mathrm{~K}$, both of the two correlation coefficients of the fitted storage modulus, and loss modulus equation are above 0.99, the results fitted well. Fitting results compared with experiment data at $293 \mathrm{~K}$ are illustrated in Figure 11.

4.3.3. Model Applicability Analysis. We may see that both of the fifth-order GM model and FD model can characterize the viscoelasticity of PVB at glass transition area accurately and the accuracy of model increases with the temperature close to $t_{g}$. When temperature is far away from the glass transition area, deviation between fitting result and experiment data increases gradually. On the other hand, this temperature range covers the most commonly used temperature of automotive windshield and architecture glazing, so both two models are suitable to be used related engineering application research.

For fifth GM model, more parameters are employed while the value of correlation coefficients between fitted and experiment result is higher than FD model in high energy absorption state, so GM model can fit the experiment result more accurate in this state. In FD model, fewer parameters are used and variable " $r$ " is employed to describe the viscoelasticity $\tan \delta$. Consequently, in future theoretical research and numerical simulation, fifth GM model can be employed to calibrate a more accurate dynamic mechanical behavior with the unlimited parameter number, while FD model is more suitable to describe the viscoelasticity of PVB in different temperatures by using fewer parameters.

\section{Concluding Remarks}

Systematic investigation of viscoelasticity of PVB based on DMA method is carried out and time/temperature effect is thoroughly studied in PVB's mechanical behavior. When PVB is in glassy and rubbery state, variation of temperature and vibration frequency causes little variation of dynamic 
modulus and PVB shows significant brittleness and viscosity, respectively, corresponding to higher dynamic modulus $\left(10^{2}\right.$ $\left.10^{3} \mathrm{MPa}\right)$ and lower dynamic $\left(10^{0}-10^{1} \mathrm{MPa}\right)$ modulus. At temperature range from $273 \mathrm{~K}$ to $323 \mathrm{~K}$, PVB is in glass transition region while the dynamic modulus varies with the temperature and frequency significantly. Relationship between $t_{g}$ and heating rate, as well as scanning frequency, is fitted. It is shown that at the heating rate from 0 to $12.5 \mathrm{~K} / \mathrm{min}$ and scanning frequency from $1 \mathrm{~Hz}$ to $50 \mathrm{~Hz}, t_{g}$ varies from $298 \mathrm{~K}$ to $319 \mathrm{~K}$. PVB has higher efficiency of energy dissipation in its high energy absorption state (at temperature $293 \sim 323 \mathrm{~K}$ ), which is more appropriate to be an energy absorption candidate as it can play a critical role in the energy mitigation behavior of laminated glass.

Viscoelasticity constitutive behavior of PVB is further discussed. PVB is proven as a thermorheological simplified material and WLF equation is fitted well with the calculated result. Dynamic modulus-frequency master curve at temperature $293 \mathrm{~K}$ is obtained based on WLF equation to characterize the dynamic modulus at frequency range from $10^{-6}$ to $10^{8} \mathrm{~Hz}$, while the transition frequency threshold value from glassy to rubbery state is gained in the master curve. GM model and FD model are employed to fit the constitutive relationship of PVB, respectively. Both fifth order GM model and FD model can characterize the viscoelasticity of PVB at glassy transition area. It is shown that in future theoretical research and numerical simulation, GM model can fit the experiment result more accurate in high energy absorption state by more parameters, while FD model is better to describe the viscoelasticity of PVB in different temperatures with fewer parameters.

This study provides important results of viscoelastic behaviors of PVB under different time and temperature situation and gives reliable models to describe the TimeTemperature-Superposition characteristics and constitutive relations of PVB. Results lay important foundations for the further researches on viscoelasticity of PVB material and the energy absorption ability of laminated glass.

\section{Conflict of Interests}

The authors declare that there is no conflict of interests regarding the publication of this paper.

\section{Acknowledgments}

The work is supported by National Natural Science Foundation of China 11372164, National Natural Science Foundation of China 11102099, and International Joint Research Project sponsored by Tsinghua University (20121080050).

\section{References}

[1] D. Otte, M. Jänsch, and C. Haasper, "Injury protection and accident causation parameters for vulnerable road users based on German In-Depth Accident Study GIDAS," Accident Analysis and Prevention, vol. 44, no. 1, pp. 149-153, 2012.
[2] J. Xu and Y. Li, "Crack analysis in PVB laminated windshield impacted by pedestrian head in traffic accident," International Journal of Crashworthiness, vol. 14, no. 1, pp. 63-71, 2009.

[3] J. Xu, Y. Li, G. Lu, and W. Zhou, "Reconstruction model of vehicle impact speed in pedestrian-vehicle accident," International Journal of Impact Engineering, vol. 36, no. 6, pp. 783-788, 2009.

[4] B. Liu, Y. Sun, Y. Li, Y. Wang, D. Ge, and J. Xu, "Systematic experimental study on mechanical behavior of PVB (polyvinyl butyral) material under various loading conditions," Polymer Engineering and Science, vol. 52, no. 5, pp. 1137-1147, 2012.

[5] S. Zhao, L. R. Dharani, L. Chai, and S. D. Barbat, "Analysis of damage in laminated automotive glazing subjected to simulated head impact," Engineering Failure Analysis, vol. 13, no. 4, pp. 582-597, 2006.

[6] J. Xu, Y. Sun, B. Liu et al., "Experimental and macroscopic investigation of dynamic crack patterns in PVB laminated glass sheets subject to light-weight impact," Engineering Failure Analysis, vol. 18, no. 6, pp. 1605-1612, 2011.

[7] G. Herndon, K. Allen, A. Roberts, D. Phillips, and S. A. Batzer, "Automotive side glazing failure due to simulated human interaction," Engineering Failure Analysis, vol. 14, no. 8, pp. 17011710, 2007.

[8] M. Wingren, Windscreen Study Usinga Free Moving Headform. An Investigation of Windcreen Behaviourwhen Subjected to Headform Impact, University of Skovde, 2011.

[9] T. Pyttel, H. Liebertz, and J. Cai, "Failure criterion for laminated glass under impact loading and its application in finite element simulation," International Journal of Impact Engineering, vol. 38, no. 4, pp. 252-263, 2011.

[10] D. Alhazov and E. Zussman, "Study of the energy absorption capabilities of laminated glass using carbon nanotubes," Composites Science and Technology, vol. 72, no. 6, pp. 681-687, 2012.

[11] R. Iwasaki, C. Sato, J. L. Latailladeand, and P. Viot, "Experimental study on the interface fracture toughness of PVB (polyvinyl butyral)/glass at high strain rates," International Journal of Crashworthiness, vol. 12, no. 3, pp. 293-298, 2007.

[12] J. Xu, Y. Li, D. Ge, B. Liu, and M. Zhu, "Experimental investigation on constitutive behavior of PVB under impact loading," International Journal of Impact Engineering, vol. 38, no. 2-3, pp. 106-114, 2011.

[13] M. Z. Aşik, "Laminated glass plates: revealing of nonlinear behavior," Computers and Structures, vol. 81, no. 28-29, pp. 2659-2671, 2003.

[14] M. Z. Aşik and S. Tezcan, "A mathematical model for the behavior of laminated glass beams," Computers and Structures, vol. 83, no. 21-22, pp. 1742-1753, 2005.

[15] P. A. Hooper, B. R. K. Blackman, and J. P. Dear, “The mechanical behaviour of poly(vinyl butyral) at different strain magnitudes and strain rates," Journal of Materials Science, vol. 47, no. 8, pp. 3564-3576, 2012.

[16] D. Callewaert, J. Belis, D. Delincé, and R. Van Impe, "Experimental stiffness characterisation of glass/ionomer laminates for structural applications," Construction and Building Matials, vol. 37, pp. 685-692, 2012.

[17] Y. Zhang, R. D. Adams, and L. Da Silva, "A rapid method of measuring the glass transition temperature using a novel dynamic mechanical analysis method," Journal of Adhesion, vol. 89, no. 10, pp. 785-806, 2013.

[18] X. Y. Shi, W. N. Bi, and S. G. Zhao, "DMA analysis of the damping of ethylene-vinyl acetate/acrylonitrile butadiene rubber blends," Journal of Applied Polymer Science, vol. 124, no. 3, pp. 2234-2239, 2012. 
[19] W. Stark, "Investigation of the curing behaviour of carbon fibre epoxy prepreg by Dynamic Mechanical Analysis DMA," Polymer Testing, vol. 32, no. 2, pp. 231-239, 2013.

[20] X. L. Zhan, Research on the Viscoelastic Properties of Asphalt Using DMA, Harbin Institute of Technology, Harbin, China, 2007.

[21] S. Y. Chen, M. Xiao, S. Wang, and Y. Meng, "Mechanical properties of block poly(propylene carbonate-cyclohexyl carbonate) investigated by nanoindentation and DMA methodologies," Journal of Applied Polymer Science, vol. 128, no. 3, pp. 1979-1986, 2013.

[22] J. J. Aklonis and W. J. MacKnight, Eds., Introduction to Polymer Viscoelasticity, Wiley-Interscience, 1983.

[23] B. H. Liu, J. Zhou, Y. Sun, Y. Wang, J. Xu, and Y. Li, "An experimental study on the dynamic viscoelasticity of PVB film material for vehicles," Automotive Engineering, vol. 34, no. 10, pp. 898-904, 2012.

[24] T. D. Gillespie, Fundamentals of Vehicle Dynamics, SAE No. R114, 1992.

[25] R. F. Chi, Concept Body Intelligentized CAE Analyse-Modal Analyse, Jilin University, jilin china, 2007.

[26] M. L. Guo, Dynamic Mechanical Thermal Analysis of Polymer and Composite Material, Chemical Industry Press, Beijing, China, 2002.

[27] C. Louter, J. Belis, F. Veer, and J.-P. Lebet, "Structural response of SG-laminated reinforced glass beams; experimental investigations on the effects of glass type, reinforcement percentage and beam size," Engineering Structures, vol. 36, pp. 292-301, 2012.

[28] Y. M. Yin, Research on Dynamic Viscoelastic Characteristics and Shear Modulus Predicting Methods for Aspgalt Mixtures Based on Dynamic Mechanical Analysis(DMA) Means, South China University of Technology, Guangzhou, China, 2010. 

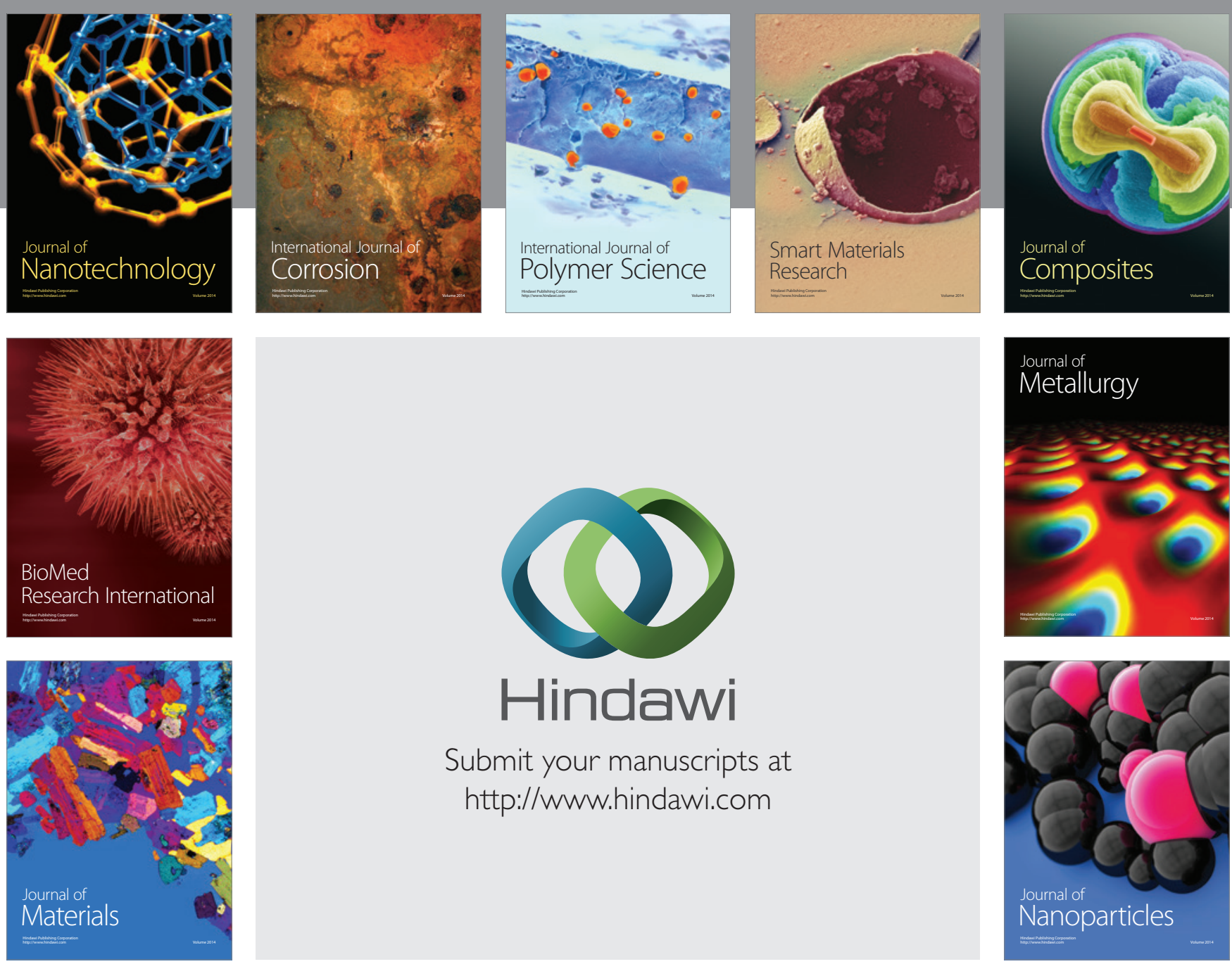

Submit your manuscripts at http://www.hindawi.com
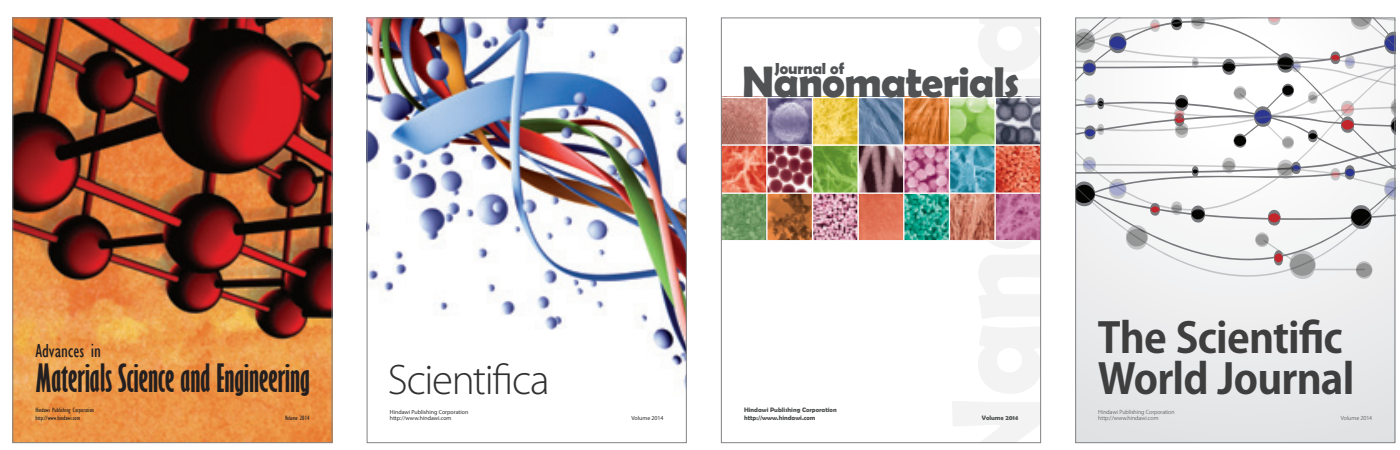

\section{The Scientific World Journal}
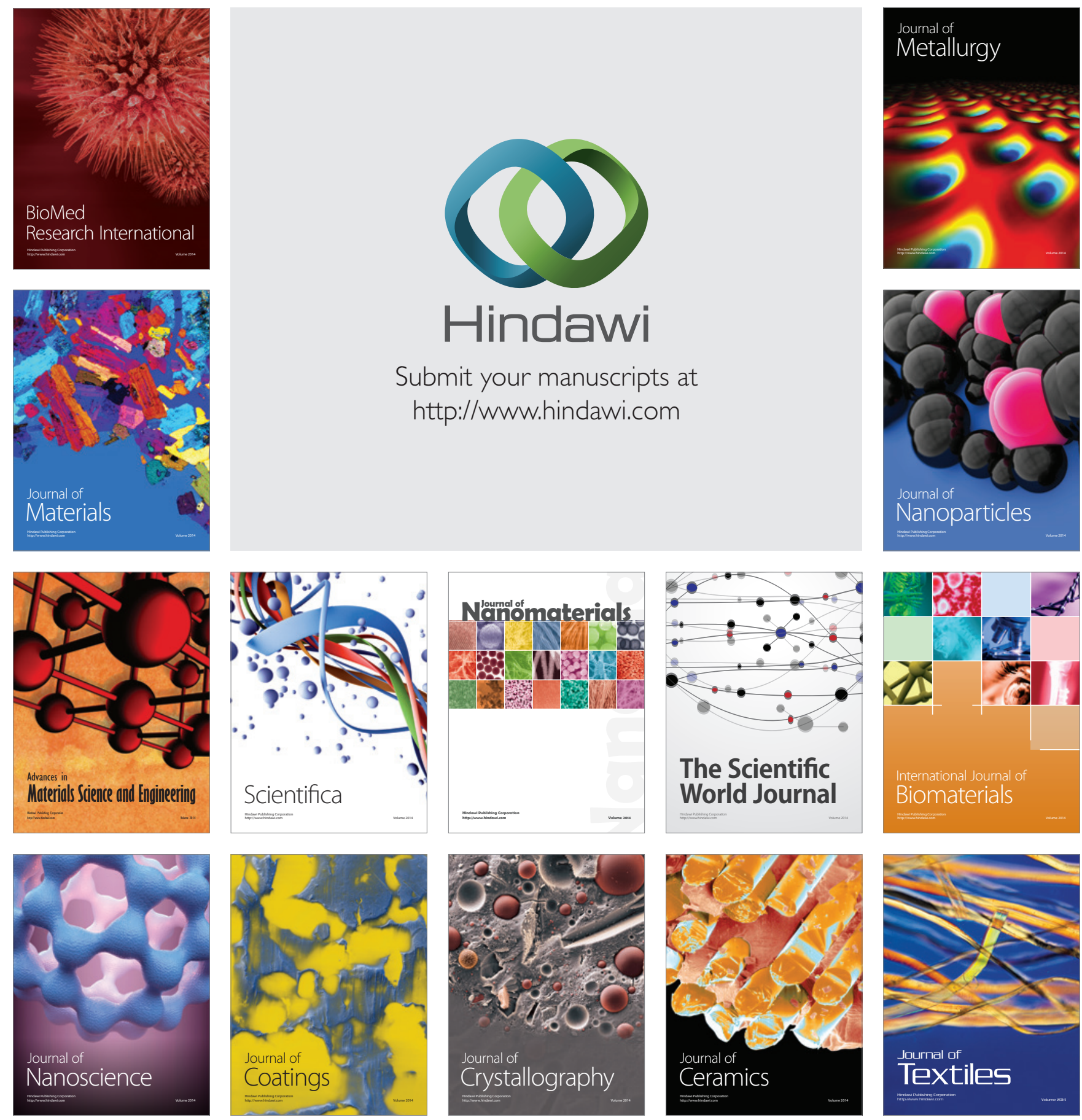